\title{
Correction to: Negative prognostic outcomes of percutaneous transhepatic biliary drainage in distal cholangiocarcinoma: a retrospective analysis using propensity score matching
}

\author{
Yutaro Matsunaga ${ }^{1} \cdot$ Ryota Higuchi $^{1}$ (1) - Takehisa Yazawa ${ }^{1}$. Shuichiro Uemura ${ }^{1}$. Wataru Izumo ${ }^{1}$ Takehiro Ota ${ }^{1}$. \\ Toru Furukawa $^{2} \cdot$ Masakazu Yamamoto $^{1}$
}

Published online: 20 July 2021

(C) Japan Society of Clinical Oncology 2021

\section{Correction to: International Journal of Clinical Oncology https://doi.org/10.1007/s10147-021-01926-8}

The original article has been published with incorrect Supplementary Figure 2 . The correct version of Supplementary Figure 2 is given in this correction.

Supplementary Information The online version contains supplementary material available at https://doi.org/10.1007/s10147-021-01975-z.

Publisher's Note Springer Nature remains neutral with regard to jurisdictional claims in published maps and institutional affiliations.

The original article can be found online at https://doi.org/10.1007/ s10147-021-01926-8.

Ryota Higuchi

higuchi.ryota@twmu.ac.jp

1 Department of Surgery, Institute of Gastroenterology, Tokyo Women's Medical University, 8-1 Kawada-cho, Shinjuku-ku, Tokyo 162-8666, Japan

2 Department of Investigative Pathology, Tohoku University Graduate School of Medicine, 2-1 Seiryomachi, Aoba-ku, Sendai 980-8575, Japan 